\title{
Thrust Augmentation and Vortex Ring Evolution in a Fully Pulsed Jet
}

\author{
Paul S. Krueger* \\ Southern Methodist University, Dallas, Texas 75275 \\ and \\ M. Gharib ${ }^{\dagger}$ \\ California Institute of Technology, Pasadena, California 91125
}

\begin{abstract}
The time-averaged thrust of an incompressible fully pulsed jet containing a period of no flow between pulses is studied experimentally as a function of pulsing duty cycle $S r_{L}$ and the ratio of the ejected slug length (per pulse) to the jet diameter $L / D$. The parameter ranges investigated were $2 \leq L / D \leq 6$ and $0.1 \leq S r_{L} \leq 0.98$. Significant thrust augmentation by pulsing was observed over the entire parameter range tested, both in terms of thrust compared to an equivalent steady jet with identical mass flux, denoted $F_{\mathrm{SJ}}>1$, and in terms of thrust compared to an equivalent intermittent jet where vortex ring formation by pulsation was ignored, denoted $F_{\mathrm{IJ}}>1 . F_{\mathrm{SJ}}$ as high as $1.90(90 \%$ thrust augmentation) was observed for the smaller $L / D$ as $S r_{L}$ approached 1.0 (with larger $F_{\mathrm{SJ}}$ at lower $S r_{L}$ ). The $F_{\mathrm{IJ}}$ results, which directly measured overpressure at the nozzle exit plane developed during vortex ring formation as the mechanism responsible for thrust augmentation, showed reduced augmentation at large $L / D$ and $S r_{L}$. The $L / D$ dependence of $F_{\mathrm{IJ}}$ parallels single-pulse $\left(S r_{L}=0\right)$ results previously studied by the authors. $\mathrm{The} S r_{L}$ dependence of $F_{\mathrm{IJ}}$ was linked to the interaction of forming vortex rings with vorticity from preceding pulses using digital particle image velocimetry (DPIV) measurements of the vorticity field. DPIV also revealed that the vortex rings tended to wander off axis and disintegrate as $S r_{L}$ became sufficiently large.
\end{abstract}

\section{Introduction}

$\mathbf{T}$ HE act of imposing a fluctuating axial velocity component on jet flow to create forced or pulsed jets has been a focal point of research in jet flows for many years. Investigations regarding the effects of pulsing on jet flows have revealed that pulsing can enhance/control orderly structure in turbulent jets ${ }^{1}$ and substantially enhance jet entrainment and mixing rates. ${ }^{1-4}$ In addition, for pulsed jets with a period of no-flow between pulses, pulsing significantly alters the development and evolution of turbulence in the jet. ${ }^{4,5}$ Pulsed jets with a period of no-flow between pulses are sometimes called fully pulsed ${ }^{4}$ or fully modulated jets. ${ }^{6}$

Most of the work on pulsed jets has focused on utilizing jet unsteadiness to enhance mixing or on understanding the overall evolution of the jet flowfield to model and/or control jet turbulence. Some work, for example, that of Wilson and Paxson ${ }^{7}$ and Sarohia et al., ${ }^{8}$ has also considered pulsed jets in conjunction with ejectors to enhance thrust augmentation of ejector configurations. These efforts were able to increase the thrust augmentation of the ejector by as much as $33 \%$ when the jet is pulsed, ${ }^{7}$ the increased performance being attributed to the enhanced entrainment provided through pulsation. ${ }^{7,8}$

Very little work, however, has been done to investigate thrust augmentation from pulsing alone, without an ejector present. Some limited data for pulsing without an ejector are presented by Sarohia et al., ${ }^{8}$ who document one test with pulsing alone. This test showed increased thrust augmentation at larger pulsing amplitudes with 5\% augmentation over the steady jet case at a jet velocity pulsing amplitude of $17 \%$, the maximum tested. Even fewer data are available relating the evolution of vortical structures in the near-jet region

Received 9 April 2004; revision received 8 October 2004; accepted for publication 19 October 2004. Copyright (C) 2005 by the American Institute of Aeronautics and Astronautics, Inc. All rights reserved. Copies of this paper may be made for personal or internal use, on condition that the copier pay the $\$ 10.00$ per-copy fee to the Copyright Clearance Center, Inc., 222 Rosewood Drive, Danvers, MA 01923; include the code 0001-1452/05 \$10.00 in correspondence with the CCC.

*Assistant Professor, Department of Mechanical Engineering. Member AIAA.

Professor, Graduate Aeronautical Laboratories and Bioengineering. of pulsed jets to thrust augmentation with or without ejectors, although Wilson and Paxon ${ }^{7}$ do mention that the diameter of vortex rings generated by pulsing appeared to determine the optimal size for the ejector in their experiments.

The correlation of thrust augmentation with pulsing amplitude reported by Sarohia et al. ${ }^{8}$ for pulsed jets with no ejector is encouraging, but suggests that large-amplitude pulsation may be necessary to achieve significant augmentation. By this reasoning, a fully pulsed jet may be more appropriate for maximizing augmentation by pulsing alone because it involves the maximum possible pulsing amplitude, without reverse flow between pulses. The result is a pulsed jet dominated by the unsteadiness of the flow. In particular, because the jet velocity returns to zero between each pulse, the flow at the initiation of each pulse is similar to that associated with the classical formation of a vortex ring from a piston-cylinder mechanism. ${ }^{9}$ As a result, vortex ring formation is likely a key feature of thrust augmentation by fully pulsed jets.

In recent work by the authors ${ }^{10}$ unsteady flow effects related to vortex ring formation were considered for single jet pulses issuing into quiescent fluid (starting jets), which are the fundamental unit of a fully pulsed jet. For the case of a single jet pulse, it was observed that the total impulse generated per pulse was substantially more than that due to momentum flux from the jet alone, the difference being due to overpressure at the nozzle exit plane (nozzle exit overpressure) during vortex ring formation. The effect was a strong function of the ratio of the length of fluid slug ejected during the pulse to the nozzle diameter, that is, the stroke-to-diameter ratio $L / D$. For the lowest $L / D$ tested $(L / D=2)$, the impulse due to overpressure, $I_{p}$, was as much as $42 \%$ of the total impulse per pulse, suggesting the potential for powerful thrust augmentation under repeated pulsing.

It was also observed that the vortex ring pinch off phenomenon discovered by Gharib et al. ${ }^{11}$ had an important effect on the results as $L / D$ was increased. Vortex ring pinch off occurs when $L / D$ is larger than a specific value, called the formation number $F$. For $L / D<F$, isolated vortex rings are formed with each pulse. For $L / D>F$, the vortex ring will stop forming midway through the pulse and pinch off from its generating jet in terms of entrainment of circulation. Krueger and Gharib ${ }^{10}$ showed that after the vortex ring pinches off, the remainder of the pulse (ejected as a trailing jet) 
contributes very little to $I_{p}$ because it behaves essentially as a steady jet. As a result, a maximum in the average thrust during a pulse $\overline{F_{p}}$ was observed at an $L / D$ very near the point where pinch off is first observed. This observation suggests that the way to impart the most momentum to the flow in a given amount of time is to use pulses of nondimensional size, that is, $L / D$, very near the formation number.

These results bolster the previous speculation regarding thrust augmentation in fully pulsed jets and highlight the potential impact of vortex ring formation in pulsed jets for applications where imparting impulse to the flow is a primary goal, such as propulsion or synthetic jet actuators. Applications, however, require repeated pulsing to supply impulse to the flow indefinitely. Repeated pulsing is significantly different from that of isolated starting jets in that jet pulses no longer issue into quiescent fluid. This implies the possible interaction of forming vortex rings with rings formed by previously ejected pulses, which may significantly affect the impulse per pulse and, hence, the thrust performance of the pulsed jet.

The present study seeks to extend the results of Krueger and Gharib $^{10}$ to the case of continuous pulsing. This is approached by considering the time-averaged thrust generated by a fully pulsed jet for a range of $L / D$ and pulsing frequency. Specific emphasis is placed on the relationship between the formation and evolution of vortex rings in the flow and the resulting thrust augmentation. Hot-film anemometry of the jet flow at the nozzle exit and digital particle image velocimetry (DPIV) of the vortical structures in the near jet are used to relate the thrust measurements with the near-field evolution of the jet.

\section{Experimental Setup}

The generation of a fully pulsed jet is illustrated schematically in Fig. 1, where a periodic series of finite-duration, round water jets with diameter $D$ are ejected into quiescent water. The length of the ejected pulses $L$ is defined by

$$
L \equiv \int_{0}^{t_{p}} U_{J}(\tau) \mathrm{d} \tau
$$

where

$$
U_{J}(t) \equiv \frac{1}{A} \int_{A} u_{J}(r, t) \mathrm{d} S=\left\langle u_{J}\right\rangle
$$

Here, $u_{J}$ is the jet velocity at the nozzle exit plane $(x=0), A$ is the cross-sectional area of the nozzle at $x=0, t_{p}$ is the pulse duration, and the brackets in Eq. (2) denote the spatial average over the nozzle exit plane. The pulse ejection rate or pulsing frequency is $f=1 / T$. A fully pulsed jet requires that $f<1 / t_{p}$ because the flow must return to zero between pulses. The parameters $f$ and $L$ can be varied independently, but the functional form of $U_{J}(t)$ during pulse ejection (also called the velocity program for the pulses) is coupled to $L$ through Eq. (1). The flow reduces to a starting jet in the limit $f \rightarrow 0$.

The dimensionless parameters characterizing the kinematics of this jet are the normalized velocity program $U_{J} / U_{\max }$ (considered as a function of $\left.t / t_{p}\right)$; the dimensionless pulse size $L / D$, that is,

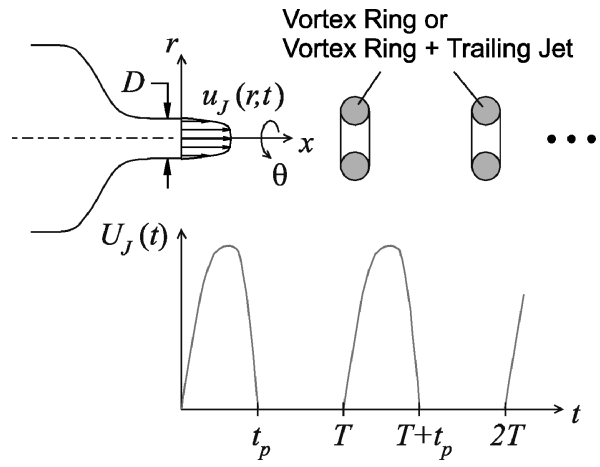

Fig. 1 General schematic of experiment.

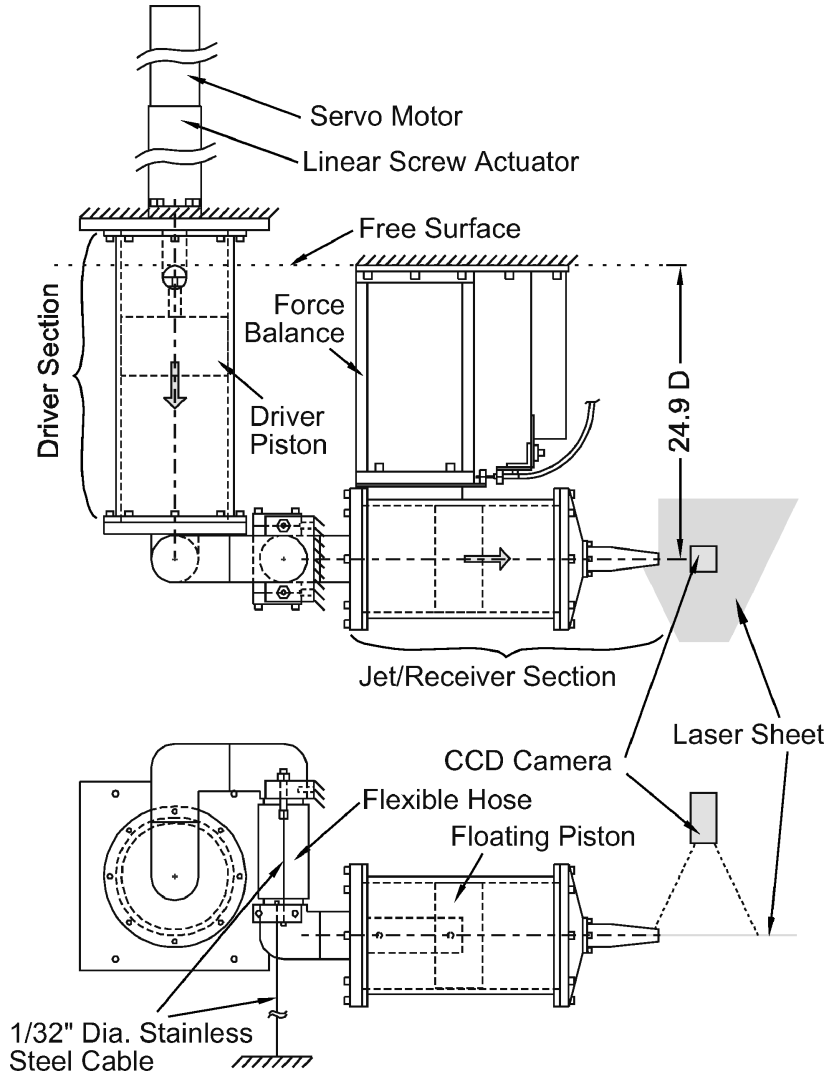

Fig. 2 Schematic of apparatus used to generate fully pulsed jet; only those hidden features necessary to illustrate the operation of the device shown.

the stroke-to-diameter ratio for a pulse; and the dimensionless frequency $S r_{L}$. $U_{\max }$ is the maximum $U_{J}(t)$ achieved during a pulse. The dimensionless frequency $S r_{L}$ is defined as

$$
S r_{L} \equiv f L / \tilde{U}_{J}
$$

where the tilde over $U_{J}$ denotes the time average only over the pulse duration $t_{p}$. Explicitly, $\tilde{U}_{J}=L / t_{p}$, from which it follows that $S r_{L}$ is equivalent to the duty cycle $t_{p} / T$. $S r_{L}$ varies between 0 and 1 for a fully pulsed jet and determines the separation between pulses.

The experimental setup used to generate a fully pulsed jet as just described is illustrated schematically in Fig. 2. The basic system is the same as that used by Krueger and Gharib. ${ }^{10}$ It consisted of two piston-cylinder systems (one oriented vertically and the other oriented horizontally) submerged in water and connected by a combination of PVC piping and a flexible hose. Because of the incompressibility of water, the floating piston in the horizontal/receiver cylinder followed the motion of the driver piston (actuated by the servomotor), as indicated by the gray arrows in Fig. 2 . The relatively large volume in the receiver cylinder allowed for more than $15 \mathrm{~s}$ of continuous pulsing for the parameters used in this investigation. The computer controlled servomotor provided direct control over $f, L$, and $U_{J}(t)$. A sharp cone angle of $7 \mathrm{deg}$ was used at the nozzle exit, as shown in Fig. 3, to promote clean vortex ring formation during pulsing and to minimize any interaction between the fully pulsed jet and the large-diameter cylindrical plenum feeding the nozzle.

The entire apparatus was mounted in a tank facility that had a steel frame and glass walls for flow visualization. The hatch marks in Fig. 2 indicate the portions of the apparatus that were, in some way, rigidly fixed to the tank frame. The minimum separation of the nozzle from any of the boundaries was the distance between the nozzle center line and the free surface, namely, $12.45 \mathrm{in} .(31.6 \mathrm{~cm}=24.9 \mathrm{D}$ where $D=0.500 \mathrm{in}$. is the nozzle diameter).

The receiver cylinder was mounted to a force balance for direct measurement of the thrust generated by the fully pulsed jet. The force balance was custom designed with a stiffness in the direction 


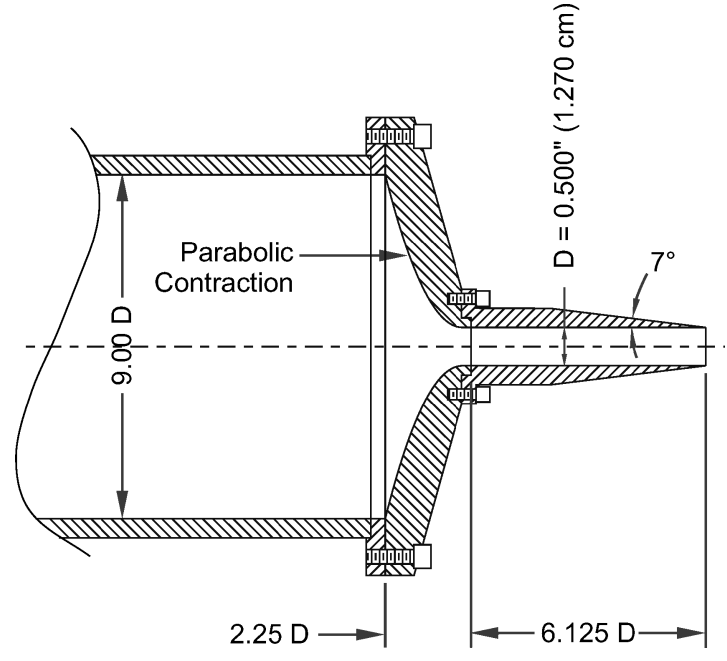

Fig. 3 Cross section of jet nozzle.

of the jet axis of $5.07 \times 10^{5} \mathrm{~N} / \mathrm{m}$ and a force resolution of better than $0.0098 \mathrm{~N}$ in the absence of any signal conditioning. The combination of a stiff force measurement system and a receiver section isolated from the driving mechanism (reducing its overall mass) resulted in a relatively high resonance frequency for the combined receiver/force balance system and no observable motion of the receiver section during pulsing.

The force measurement system did, however, show a noticeable linear drift in the zero point during pulsing due to hysteresis in the system. The zero-point drift was removed from the measurements in the postprocessing phase. The accuracy of the time-averaged thrust measurements obtained in this way was verified independently for several cases by measuring the time-averaged force on a $30.5 \times 30.5 \mathrm{~cm}$ plate placed $12 \mathrm{D}$ downstream of the nozzle and oriented normal to the jet stream. The nominal values for the corrected force balance measurements and the plate measurements agreed to within $4 \%$, which was within the uncertainty of the plate measurements. Nevertheless, the corrected force balance measurements still showed a random variation of a few percent for tests at the same conditions. This variation proved to be the dominant source of error in the thrust measurements. An estimate of the error was determined from the standard deviation of the mean for five or more tests at the same conditions. Only results from the force balance measurements will be presented.

Measurements of the flow structure in the near-jet region were made using DPIV. For these measurements, a laser sheet and chargecoupled device (CCD) camera were mounted as shown in Fig. 2. The flow was seeded with neutrally buoyant, silver-coated hollow glass spheres with diameters in the range of $20-50 \mu \mathrm{m}$. The particles were illuminated with an Nd:YAG laser whose beam was formed into a 2-mm-thick sheet using a cylindrical lens. The particle images were recorded on a $480 \times 768 \mathrm{CCD}$. By the use of a cross-correlation algorithm based on that implemented by Willert and Gharib, ${ }^{12}$ the images were processed with a $32 \times 32$ interrogation window at $75 \%$ overlap. Processing a second time using a $16 \times 16$ interrogation window at $50 \%$ overlap with a window shifting algorithm ${ }^{13}$ produced flow vector fields with a spatial resolution of $0.097 \mathrm{D}$ in $x$ and $0.11 D$ in $r$ in the downstream region $0 \leq x / D \leq 9$.

The jet velocity at the nozzle exit plane $(x=0)$ was measured with a TSI (TSI, Inc.) $1231 \mathrm{~W}$ hot-film probe using a TSI IFA-100 flow analyzer and a Model 150 anemometer. The probe was placed at the jet centerline and calibrated by moving the driver piston at several steady velocities, giving a calibration for the mean velocity at the nozzle exit plane $U_{J}$. A calibration for the centerline velocity $u_{\mathrm{cl}}=\left.u_{J}\right|_{r=0}$ was also obtained from the relation between $u_{\mathrm{cl}}$ and $U_{J}$ measured using DPIV (for steady commanded piston velocities). Hot-film measurements during pulsing provided measurements of $U_{J}(t)$ and $u_{\mathrm{cl}}(t)$ for the range of $L$ and $f$ tested in this investigation. To avoid any effect the presence of the hot-film might have on the thrust measurements, jet velocity measurements were made immediately before and after experiments measuring thrust.

\section{Definition of Thrust Augmentation}

To determine whether thrust augmentation has been achieved by pulsing, it is necessary to compare the measurements of the timeaveraged thrust from the fully pulsed jet $\overline{F_{T}}$ with the thrust from an equivalent jet for which the effects of pulsing are not present. Perhaps the simplest case for comparison is a steady jet with the same time-averaged mass flux as the fully pulsed jet. The thrust for such a jet is given by

$$
F_{s}=\rho A U_{s}^{2}
$$

where the matching constraint on mass flux requires

$$
U_{s}=\left(t_{p} / T\right) \tilde{U}_{J}=S r_{L} \widetilde{\left\langle u_{j}\right\rangle}
$$

The quotient of $\overline{F_{T}}$ with $F_{s}$ gives an augmentation ratio we designate the steady-jet normalization, namely,

$$
F_{\mathrm{SJ}} \equiv \overline{F_{T}} / F_{s}
$$

This is the same ratio used by Sarohia et al. ${ }^{8}$ for defining thrust augmentation of a pulsed jet (with or without an ejector). If any thrust augmentation is achieved by pulsing, $F_{\mathrm{SJ}}$ will be greater than one.

Although the steady-jet normalization is intuitive and practical, it bears little relation to the flow physics expected to be responsible for any thrust augmentation achieved, namely, additional thrust provided by nozzle exit overpressure developed during vortex ring formation at each pulse. To highlight the effects of vortex ring formation on thrust augmentation, we compare $\overline{F_{T}}$ with a hypothetical jet of identical $f, L$, and $U_{J}(t)$, but for which all effects of vortex ring formation have been ignored. Conceptually, such a jet can be visualized as a steady jet chopped into segments of length $L$, for which reason we denote this hypothetical case as an intermittent jet. For the intermittent jet, no nozzle exit overpressure is developed, and its time-averaged thrust is

$$
\overline{F_{\mathrm{IJ}}} \equiv \frac{\rho}{T} \int_{0}^{t_{p}} \int_{A} u_{J}^{2}(r, \tau) \mathrm{d} S \mathrm{~d} \tau=\rho A \operatorname{Sr}_{L} \widetilde{\left\langle u_{J}^{2}\right\rangle}
$$

The quotient of $\overline{F_{T}}$ with $\overline{F_{\mathrm{IJ}}}$ provides a second augmentation ratio dubbed the intermittent-jet normalization, namely,

$$
F_{\mathrm{IJ}} \equiv \overline{F_{T}} / \overline{F_{\mathrm{IJ}}}
$$

This normalization provides a direct measure of the contribution of nozzle exit overpressure to $\overline{F_{T}}$. In particular, for $f \rightarrow 0$, it reduces to $F_{\mathrm{IJ}}=I / I_{U}$, where $I$ is the total impulse generated during a single pulse and $I_{U}=\rho A t_{p} \widetilde{\left\langle u_{J}^{2}\right\rangle}$ is the impulse delivered by the jet momentum flux during a pulse. Thus, $F_{\mathrm{JJ}}$ also provides a way to directly compare the single-pulse results of Krueger and Gharib ${ }^{10}$ with the results of this investigation.

Whereas both thrust normalizations provide important and unique perspectives on the problem of thrust augmentation, there is a simple relationship between them. Specifically,

$$
F_{\mathrm{SJ}}=S\left(F_{\mathrm{IJ}} / S r_{L}\right)
$$

where

$$
S \equiv \widetilde{\left\langle u_{J}^{2}\right\rangle} / \tilde{U}_{J}^{2}
$$

is a shape factor for the velocity program $U_{J}(t)$. Because $F_{\mathrm{IJ}}$ approaches the nonzero value of $I / I_{U}$ as $S r_{L}$ (and $f$ ) approaches zero, $F_{\mathrm{SJ}}$ becomes unbounded as the pulsing frequency is reduced. This undesirable dependence obscures much of the interesting behavior in the results, and so the following presentation will focus on $F_{\mathrm{IJ}}$. 


\section{Measurements of Thrust Augmentation}

Two classes of velocity programs were used to generate the pulses in the fully pulsed jets for a range of $L / D$ and $S r_{L}$. Hot-film anemometry measurements of the velocity programs for each $L / D$ in each class (averaged over 20 realizations at a pulsing frequency of $2 \mathrm{~Hz})$ are shown in Fig. 4. The $U$ in the $U_{J}(t) / U$ normalization for the ordinate represents the desired peak velocity for the programs, namely, $1.03 \mathrm{~m} / \mathrm{s}$ for Fig. $4 \mathrm{a}$ and $0.74 \mathrm{~m} / \mathrm{s}$ for Fig. $4 \mathrm{~b}$. Both classes have a generally negative sloping character in that the peak in $U_{J}(t) / U$ occurs at $t / t_{p}<0.5$, that is, the jet accelerates rapidly during pulse initiation and then decelerates more slowly as the pulse terminates. Hence, the velocity programs in Fig. 4a are designated the negative sloping (NS) ramps and those in Fig. 4b the NS2 ramps. Ideally, the velocity programs in Fig. 4 would be identical for each $L / D$, but a small amount of elasticity between the driver and floating pistons prevented this ideal from being attained in the present investigation. Nevertheless, the overall form of the velocity programs in each class is as described. Following Krueger and Gharib ${ }^{10}$ and Rosenfeld et al., ${ }^{14}$ the Reynolds number for these velocity programs is defined as

$$
R e_{m}=\left(U_{\max } D\right) / v
$$

The Reynolds number $R e_{m}$ for the NS ramps is $1.3 \times 10^{4}$ to within $9 \%$ and is $9.1 \times 10^{3}$ to within $3 \%$ for the NS2 ramps.

The NS ramps for this investigation are identical to the NS ramps used by Krueger and Gharib, ${ }^{10}$ making the fully pulsed jets with NS ramps a direct extension of their single-pulse results. The NS2 ramps are introduced in this experiment to confirm the NS ramp results at a lower Reynolds number and broader $S r_{L}$ range.

Other types of velocity programs are possible. For instance, Krueger and Gharib ${ }^{10}$ also considered positive sloping velocity programs [with peak $U_{J}(t) / U$ at $t / t_{p}>0.5$ ] to illustrate the effects
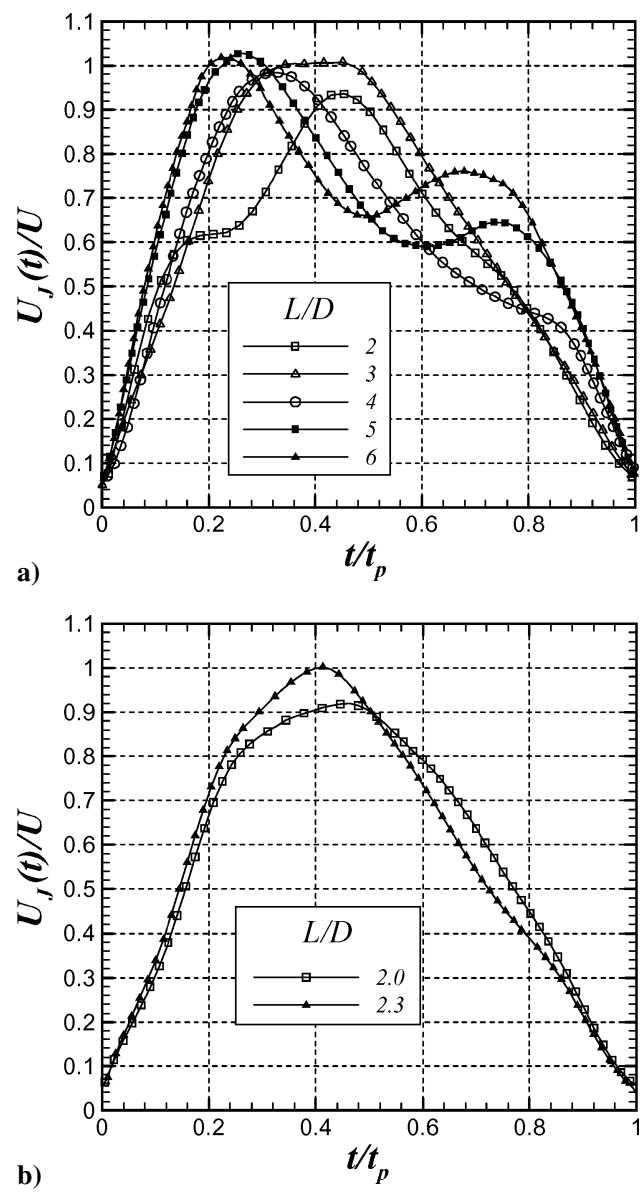

Fig. 4 Velocity programs used in fully pulsed jets: a) NS and b) NS2 ramps. of formation number on the impulse and nozzle exit overpressure associated with individual pulses because vortex ring pinch off was delayed for the positive sloping cases. Because the qualitative behavior of the NS and positive sloping cases is similar in the singlepulse case, only the generally NS velocity programs in Fig. 4 will be considered here.

Fully pulsed jets were generated using the NS and NS2 ramp velocity programs, with $2.0 \leq L / D \leq 6.0$ for the NS ramps, nominally in increments of $1.0 L / D$, and with $L / D=2.0$ and 2.3 (nominally) for the NS2 ramps. The frequency ranges corresponded to $0.1<S r_{L}<0.85$ for the NS ramps (with $S r_{L}$ up to 0.97 for $L / D=2.0$ ) and $0.1<S r_{L}<0.98$ for the NS2 ramps in increments of approximately 0.05 . For each combination of $L / D$ and $S r_{L}$, the time average of the measured thrust $\overline{F_{T}}$ was determined by computing the running average of the measured thrust and taking the mean value to which the running average had converged by the end of the test. To ensure good convergence of the running average, tests long enough to include at least 20 pulses were used. To avoid the effect of startup transients, the first $1.0 \mathrm{~s}$ of thrust measurements were not included in the evaluation of $\overline{F_{T}}$. To determine the error associated with hysteresis effects discussed in Sec. II, a minimum of five tests were performed at each condition (more at lower $S r_{L}$ ) where the standard deviation of the mean for $\overline{F_{T}}$ was taken as the error estimate for the time-averaged thrust.

To determine $F_{\mathrm{IJ}}$ and $F_{\mathrm{SJ}}$, measurements of $\widetilde{\left\langle u_{J}^{2}\right\rangle}, \tilde{U}_{J}$, and $t_{p}$ were obtained from the hot-film measurements of the jet velocity over the frequency range tested at each $L / D$ for the NS and NS2 ramps. To obtain accurate measurements of $\left\langle u_{J}^{2}\right\rangle$, the velocity profile $u_{J}(r, t)$ was estimated by assuming a parabolic profile in the boundary layer and uniform flow outside the boundary layer in the jet core. The boundary-layer thickness was estimated by comparing the hot-film measurements of $U_{J}(t)$ with $u_{\mathrm{cl}}(t)$ under the assumed shape for the velocity profile. This technique proved to be accurate to within 5\% (Ref. 10). Measurements of $\tilde{U}_{J}$ and $t_{p}$ were obtained directly from hot-film measurements of $U_{J}(t)$, where $t_{p}$ was defined as the period over which $U_{J}(t)$ is greater than $5 \%$ of $U_{\max }$.

Some variation in $\left\langle u_{J}^{2}\right\rangle, \tilde{U}_{J}, t_{p}$, and $L$ as a function of frequency was detected, even though the commanded piston motion was not altered with frequency. For example, variations in $L / D$ were within \pm 0.2 from the nominal value with lower values occurring at lower pulsing frequencies. The variations stemmed from the same system flexibility between the driver and floating piston that prevented the velocity programs from collapsing to the same curve for all $L / D$ in Fig. 4. The effect of these variations on $\overline{F_{T}}$ was factored out of the thrust augmentation measurements by using the frequencydependent measurements of $\left\langle u_{J}^{2}\right\rangle, \tilde{U}_{J}$, and $t_{p}$ when computing $F_{\mathrm{IJ}}$, $F_{\mathrm{SJ}}$, and $S r_{L}$

Using the aforementioned methodology for the NS ramps gives the $F_{\mathrm{IJ}}$ results shown in Fig. 5. The single pulse $\left(S r_{L}=0\right)$ results from Krueger and Gharib ${ }^{10}$ are included in Fig. 5 for comparison. The uncertainty in the measurements of $F_{\mathrm{IJ}}$ fall between \pm 0.02 and \pm 0.06 , with the lowest uncertainty occurring for large $S r_{L}$ at large $L / D$ and vice versa.

Several important conclusions follow from the $F_{\mathrm{IJ}}$ results. First, $F_{\mathrm{IJ}}$ is greater than one for all conditions tested. The lowest $F_{\mathrm{IJ}}$ in Fig. 5 is 1.20, indicating that the time-averaged thrust is more than $20 \%$ greater than the thrust expected from jet momentum flux alone and can be as much as $90 \%$ greater than the jet momentum flux (for $L / D=2$ and $S r_{L}=0.15$ ). Thus, pulsing provides substantial thrust augmentation in the sense that a significant portion of the thrust is provided by nozzle exit overpressure. Similar statements can be made for $F_{\mathrm{SJ}}$ by comparison of Fig. 5 with Eq. (9) and the values of the shape factor $S$ in Table 1. In particular, even for $S r_{L}$ near one (where the lowest $F_{\mathrm{SJ}}$ occur), $F_{\mathrm{SJ}}$ can be as much as 1.90 for $L / D=2$, indicating a $90 \%$ thrust augmentation for the fully pulsed jet over a steady jet of equivalent mass flux simply due to the highly pulsed nature of the jet. This is a dramatic improvement over the $5 \%$ thrust augmentation observed by Sarohia et al. ${ }^{8}$ for a jet velocity pulsing amplitude of $17 \%$, confirming the earlier hypothesis that maximizing pulsing amplitude with a fully pulsed jet should maximize the thrust augmentation achievable through pulsing alone. 

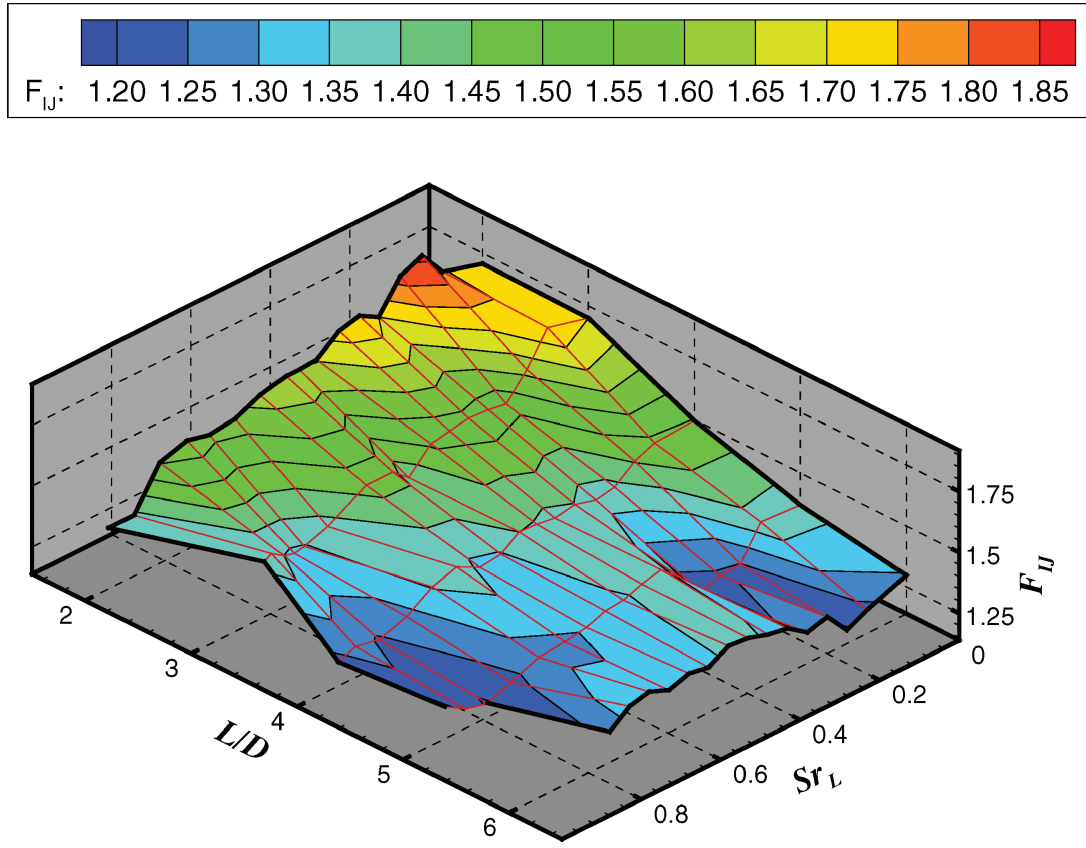

Fig. 5 Intermittent jet normalized thrust for the NS ramps.

Table 1 Values of shape factor $S$ for NS and NS2 ramps

\begin{tabular}{lcc}
\hline \hline$L / D$ (nominal) & NS ramps & NS2 ramps \\
\hline 2 & $1.30 \pm 0.03$ & $1.38 \pm 0.02$ \\
2.3 & - & $1.37 \pm 0.01$ \\
3 & $1.37 \pm 0.01$ & - \\
4 & $1.34 \pm 0.01$ & - \\
5 & $1.26 \pm 0.01$ & - \\
6 & $1.23 \pm 0.01$ & - \\
\hline
\end{tabular}

The $F_{\mathrm{IJ}}$ results also indicate that thrust augmentation appears to be more effective for smaller $L / D$. This is especially apparent at $S r_{L} \leq 0.1$, where $F_{\mathrm{IJ}}$ shows a dramatic decrease for $L / D>3$. The general predominance of thrust augmentation at low $L / D$ is in accord with the single-pulse $\left(S r_{L}=0\right)$ results of Krueger and Gharib. ${ }^{10}$ For single pulses, nozzle exit overpressure is most significant at low $L / D$ where isolated vortex rings without a trailing jet were formed by the starting jet. Krueger and Gharib ${ }^{10}$ explained this effect in terms of the additional pressure required at the nozzle exit plane during ring formation to supply impulse to the ambient fluid accelerated with the ring in the form of 1) fluid entrained into the ring and 2) the added mass of the ring, that is, fluid pushed out of the way by the ring during the formation process. These mechanisms shut down after the ring stops forming and pinches off from its trailing jet, which occurs for $L / D>3$ with the NS ramps. Hence, for larger $L / D$, nozzle exit overpressure contributes a smaller fraction to the total impulse per pulse at $S r_{L}=0$, which lowers $F_{\mathrm{IJ}}$. For the pulsed case $\left(S r_{L}>0\right)$, the general decrease in $F_{\mathrm{IJ}}$ observed as $L / D$ increases beyond three suggests that thrust augmentation is governed by vortex ring formation in this case as well, with more significant augmentation obtained when vortex ring pinch off is avoided.

Finally, Fig. 5 indicates that thrust augmentation tends to degrade with increasing $S r_{L}$. At a fixed $L / D$, a sharp drop in $F_{\mathrm{IJ}}$ with increasing $S r_{L}$ is first observed at $S r_{L}=0.1$ for $L / D \geq 3$ and at $S r_{L}=0.25$ for $L / D=2$. Following the initial drop, there is a more gradual decrease in $F_{\mathrm{IJ}}$ with increasing $S r_{L}$, the magnitude of which seems to be more substantial for smaller $L / D$. Some exceptions to this latter observation are apparent, for example, the hump in $F_{\mathrm{IJ}}$ for the midrange $S r_{L}$ at $L / D=5$ and 6 , but these variations are within the experimental uncertainty of the measurements.

Similar trends in $F_{\mathrm{IJ}}$ with increasing $S r_{L}$ appear for the NS2 ramps as well, as shown in Fig. 6. Here the switch from a rapid decline in $F_{\mathrm{IJ}}$ at low $S r_{L}$ to a more gradual decline for higher $S r_{L}$ is observed

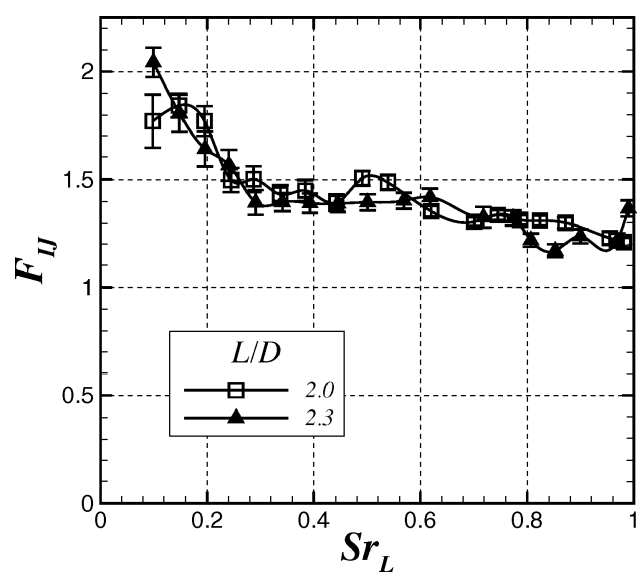

Fig. $6 F_{\mathrm{IJ}}$ results for NS2 ramps.

at around $S r_{L} \approx 0.3$ for both $L / D=2$ and 2.3. Notice also that $F_{\mathrm{IJ}}$ for the NS2 ramps is significantly larger than unity, in agreement with the NS ramp results.

The interesting dependence of $F_{\mathrm{IJ}}$ on $S r_{L}$ is due to the interaction of forming vortex rings with preceding pulses, an effect distinctly different from the single-pulse results. For the sharp decrease in $F_{\mathrm{IJ}}$ at low $S r_{L}$, careful consideration of Fig. 5 suggests that the likely cause is an interaction between the forming vortex rings and lingering remnants of vorticity from preceding pulses because the initial decrease is largest for cases of $L / D \geq 3$, which leave a trailing jet behind each vortex ring. (The trailing jets for $L / D=3$ of the NS ramps are very weak but measurable.) A trailing jet convects downstream slower than its leading vortex ring, and so it lingers near the nozzle where it may interact with the next pulse.

To investigate the interaction of a forming ring with the trailing jet of a preceding pulse, we consider the case for $L / D=2.3$ of the NS2 ramps. In this case, the gradual deceleration of the jet velocity near the end of the pulse allows a very weak trailing jet to pinch off from the primary ring, as illustrated in the DPIV measurements of azimuthal vorticity shown in Figs. 7 and 8. Figure 7a illustrates the vorticity field for $S r_{L}=0.11$, which is low enough that viscosity dissipates the trailing jet remnant before the next pulse emerges as shown in Fig. 7b. In Fig. 8, $S r_{L}=0.29$ and the small vorticity patches associated with the trailing jet remain close to the nozzle 


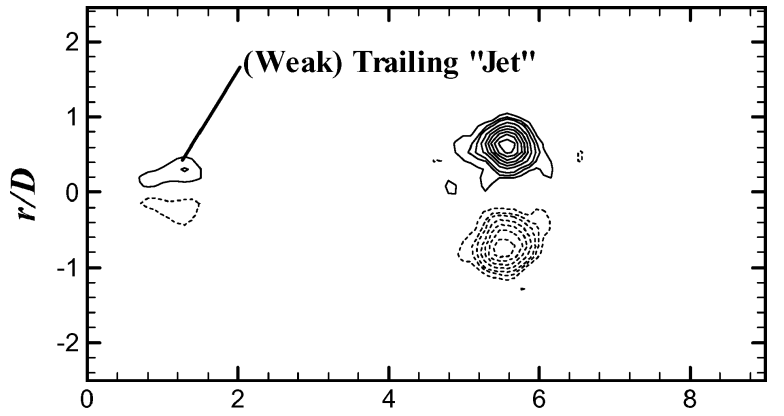

a)

$\boldsymbol{x} / \boldsymbol{D}$

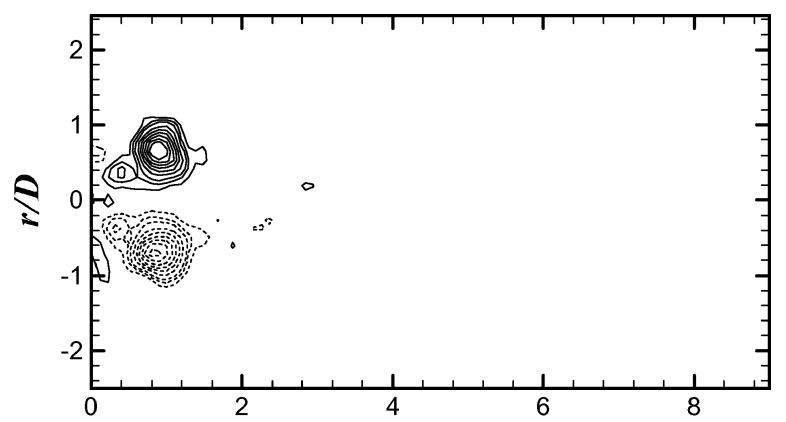

$\boldsymbol{x} / \mathrm{D}$

Contours of dimensionless azimuthal vorticity $\left(\omega_{\theta} D / U_{\text {max }}\right)$ for two instances of the $L / D=2.3$, NS2 ramp case at $S r_{L}=0.11$ : a) $t=t_{1}$ and b) $t=t_{1}+0.40 \mathrm{~s}:---$-, negative vorticity; minimum contour plotted of a given sense is 0.3 ; contour divisions are 0.3 up to 0.9 contour and 0.6 thereafter.

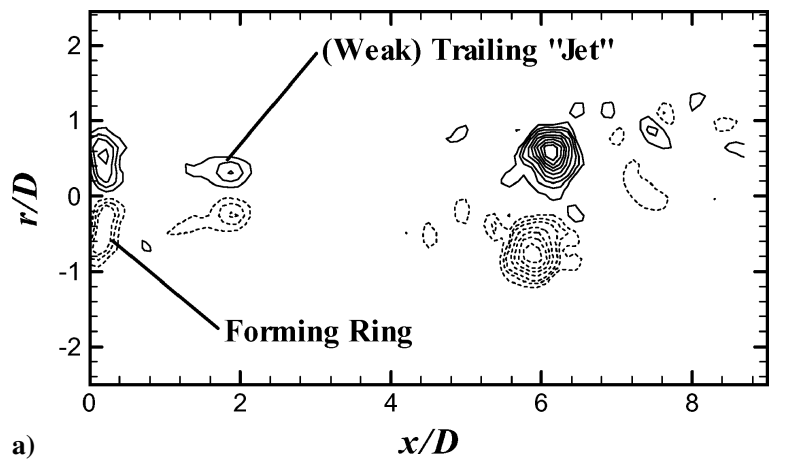

a)

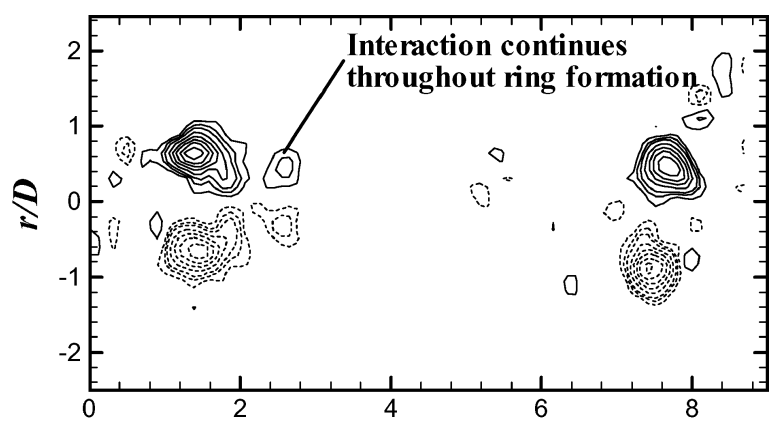

b)

$x / D$

Fig. 8 Contours of dimensionless azimuthal vorticity $\left(\omega_{\theta} D / U_{\max }\right)$ for two instances of $L / D=2.3$, NS2 ramp case at $S r_{L}=0.29$ : a) $t=t_{1}$ and b) $t=t_{1}+0.067 \mathrm{~s}$; contour levels same as Fig. 7 .

as the next pulse is emerging, thereby interacting with the forming vortex ring. Clearly, the difference between the $S r_{L}=0.11$ and 0.29 cases is the presence of trailing jet vorticity near the nozzle during ring formation for $S r_{L}=0.29$. Thus, the rapid decline in $F_{\mathrm{IJ}}$ at low $S r_{L}$ is directly related to an interaction between trailing jets and emerging pulses.

The effect of trailing jets on the initial reduction in $F_{\mathrm{IJ}}$ is supported quantitatively as well. For the NS2 ramps, $F_{\mathrm{IJ}}$ decreases by $0.27 \pm$
0.18 between $S r_{L}=0.10$ and $S r_{L}=0.29$ for $L / D=2.0$, whereas $F_{\mathrm{IJ}}$ decreases by $0.65 \pm 0.13$ over the same $S r_{L}$ range for $L / D=2.3$. Likewise, the average trailing jet circulation [determined from the DPIV data using Eq. (12)], increases from $7.2 \mathrm{~cm}^{2} / \mathrm{s}$ for $L / D=2.0$ to $12.0 \mathrm{~cm}^{2} / \mathrm{s}$ for $L / D=2.3$. Thus, the magnitude of the initial decrease in $F_{\mathrm{IJ}}$ is correlated with the strength of the trailing jet interacting with each emerging pulse. Similar behavior is apparent for the NS ramps where the initial decrease in $F_{\mathrm{IJ}}$ is more pronounced at higher $L / D$ owing to stronger trailing jets.

Physically, the initial reduction in $F_{\mathrm{IJ}}$ is related to a reduction in overpressure, which occurs because formation of the vortex rings is no longer equivalent to formation in quiescent fluid due to the presence of trailing jet vorticity. The existing fluid motion in a trailing jet has at least two effects on a forming ring. First, some of the ambient fluid already has nonzero velocity, and so an emerging jet does not have to accelerate it from rest and the overpressure required to move this fluid as the ring is forming is lower. Second, trailing jet vorticity alters the initial rollup of the shear layer in an emerging jet (when the forming ring and trailing jet have comparable circulation), which subsequently effects the overall development of the ring. Because trailing jets can be relatively weak (with circulation less than $10 \%$ of the ring circulation for the NS2 ramps), the first mechanism is likely not the primary contributor to the initial reduction in $F_{\mathrm{IJ}}$. The role of the second mechanism was more difficult to verify because the short pulse times (less than $0.07 \mathrm{~s}$ for $L / D \leq 2.3$ ) made the ring formation process impossible to resolve temporally with DPIV. Nevertheless, indirect evidence of this mechanism can be observed in measurements of vortex location for several vortex rings at different stages of formation. In particular, at higher $S r_{L}$, the vortex trajectory is altered during the initial stages of ring formation, and the final ring radius is reduced, implying a lower ring impulse and, hence, lower $F_{\mathrm{IJ}}$. These features are apparent in Fig. 9 and will be discussed in more detail hereafter.

Whereas the preceding discussion attributes the initial, abrupt decrease in $F_{\mathrm{IJ}}$ as $S r_{L}$ increases to an (unfavorable) interaction of forming vortex rings with remnants of preceding pulses, the gentle decrease in $F_{\mathrm{IJ}}$ with $S r_{L}$ that subsequently appears can be explained in terms of a more direct interaction of entire pulses. That is, as $S r_{L}$ increases, the vorticity from preceding pulses (vortex rings and trailing jets) is closer to the nozzle. An emerging ring, therefore, encounters less resistance from the ambient fluid because a greater fraction of fluid close to the nozzle already has momentum. As a result, the overpressure normally required to accelerate the ambient fluid during ring formation is less, and $F_{\mathrm{IJ}}$ decreases as $S r_{L}$ increases. The effect is rather weak, however, because $F_{\mathrm{IJ}}$ decreases by an amount in the range $0.1-0.2$ as $S r_{L}$ increases from 0.3 to 0.98 for the NS2 ramps. Such a small effect is not surprising because, as will be discussed hereafter, the vortex rings are never separated by less than four ring radii for the NS2 ramps, even at $S r_{L}=0.98$ (Fig. 10).

The two mechanisms proposed for the observed reduction in $F_{\mathrm{IJ}}$ can both be summarized as effects of ambient fluid motion from preceding pulses on vortex ring formation. The reason for distinguishing between an interaction with a trailing jet and with entire pulses is that the effects of a trailing jet appear at a much lower $S r_{L}$ than might otherwise be expected because trailing jets remain relatively close to the nozzle exit plane due to their weaker vorticity. The trailing jet interaction also has an unexpectedly strong effect given the small relative strength of trailing jets (at least for small $L / D$ ). The interaction between entire pulses, on the other hand, is relevant whether or not a trailing jet is present, but it is not expected to play a significant role until $S r_{L}$ approaches 1.0. In the case of small $L / D$, that is, $L / D<F$, this is primarily an interaction between the vortex rings.

\section{Vortex Evolution in the Near Jet}

The preceding results and discussion highlight the connection between thrust augmentation as measured by $F_{\mathrm{IJ}}$ and certain features of the vorticity in the jet. Motivated by this connection, the evolution of vorticity in the jet is now considered from a more global perspective. The focus will be on the jet behavior within nine diameters of the nozzle exit because this was the region interrogated with DPIV. 


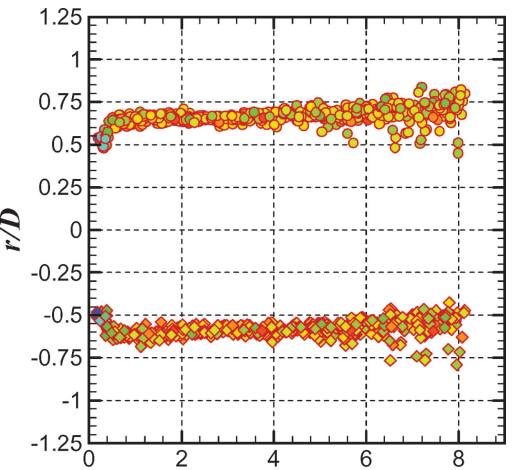

a)

$x / D$

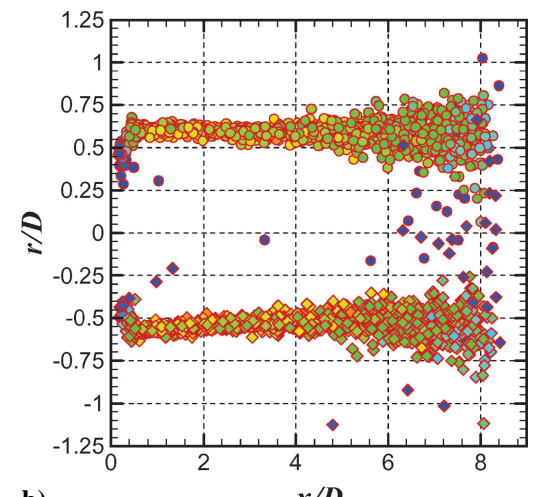

b)

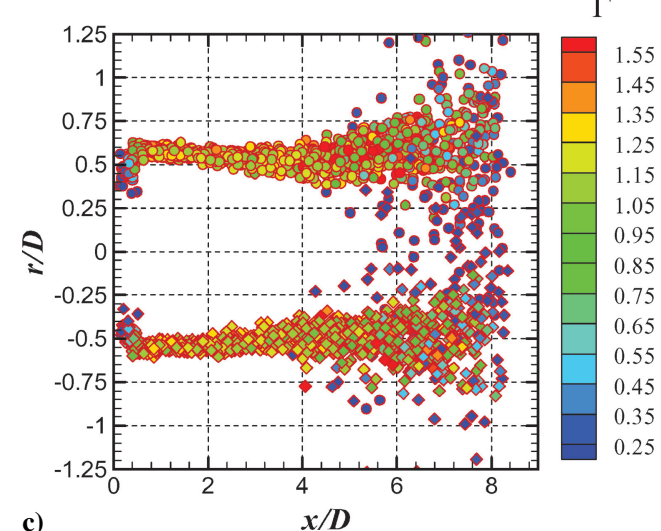

c)

Fig. 9 Centroid locations for $L / D=2.0, \mathrm{NS} 2$ ramp: $\left.\left.S r_{L}=\mathbf{a}\right) 0.13, \mathrm{~b}\right) 0.54$, and c) 0.82 ; circulation is made dimensionless according to $\Gamma^{*} \equiv \Gamma /\left(U_{\text {max }} D\right)$.

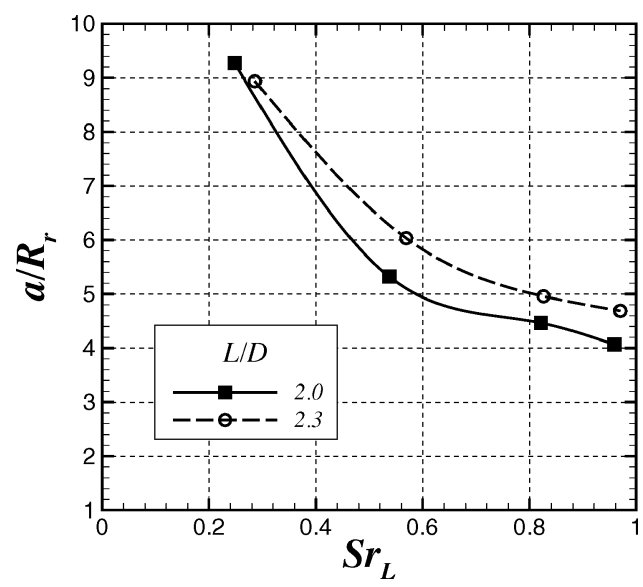

Fig. 10 Ring separation in the range $1<x / D<3$ for the NS2 ramps.

We begin by considering the simplest model of a fully pulsed jet that respects the inherently unsteady nature of the flow, namely, an infinite train of equally spaced coaxial vortex rings as illustrated in Fig. 1. Such a model was proposed by Weihs ${ }^{15}$ to describe the jet propulsion of aquatic creatures such as squid. The separation between the vortex rings is a key parameter in this model because it governs the influence of the vortex rings on each other. Weihs considered only the interaction due to the velocity induced by vortex rings on each other. Under the assumption that all other properties of the rings (such as circulation and associated mass) were held constant, the mutually induced velocity of the vortex rings would cause the total translational velocity of each vortex ring to increase as their separation decreased. Consequently, Weihs concluded that the downstream momentum flux of a fully pulsed jet would increase dramatically if the ring separation $a$ was less than about three ring radii $R_{r}$, implying substantial propulsive benefits from rapid pulsing.

For the present investigation, vortex ring separation was determined from the DPIV measurements of the vortex ring centroids in the range $1<x / D<3$. (This range was used because identifying vortex rings for $x / D>5$ became problematic at high $S r_{L}$, as will be discussed later.) The resulting measurements of the mean normalized vortex ring separation $a / R_{r}$ are shown in Fig. 10 as a function of $S r_{L}$ for the NS2 ramps. For these results, the ring radius based on vorticity centroid location $\left[r_{c}\right.$ as defined in Eqs. (13)] was taken as the ring radius $R_{r}$.

As expected, Fig. 10 shows that the ring separation decreases with increasing $S r_{L}$. Yet, $a / R_{r}$ never drops below 4.0, and the rate of decrease in $a / R_{r}$ is noticeably non-linear with $a / R_{r}$ leveling off as $S r_{L}$ approaches 1.0. Thus, it appears to be difficult to achieve the condition of $a / R_{r}<3$ necessary to observe the propulsive benefits predicted by Weihs. ${ }^{15}$ Indeed, no substantial rise in thrust augmentation was observed in the present investigation at high $S r_{L}$. Rather, as discussed earlier, augmentation decreased as $S r_{L}$ increased for a fixed $L / D$ due to an effect not considered by Weihs, namely, the influence of preexisting ambient fluid motion from previous pulses on vortex ring formation in subsequent pulses.

Modeling the jet as a series of coaxial vortex rings, however, oversimplifies the complex nature of vortex ring formation and evolution. For instance, when $L / D>F$, each pulse produces a vortex ring and a trailing jet, so that vortex rings are no longer the sole features in the jet. However, even in the case of $L / D<F$ where vortex rings dominate the flow (at least close to the nozzle), the motion and evolution of the vortex rings have some unexpected characteristics.

To investigate the global structure and evolution of the jet in a systematic way, the centroids and the total circulation associated with the vorticity contained within closed contours at a level of $20 \mathrm{~s}^{-1}$ of a given sense were cataloged from the DPIV measurements of azimuthal vorticity in the jet. (The $20 \mathrm{~s}^{-1}$ contour level was used for these data because it was just outside the inherent noise near zero vorticity.) To facilitate display of the data, only contours containing peak vorticity greater than $20 \%$ of the maximum vorticity in an image were considered. The circulation $\Gamma$ and centroid locations $\left(x_{c}, r_{c}\right)$ were determined according to the formulas

$$
\begin{gathered}
\Gamma=\int_{A_{C}} \omega_{\theta} \mathrm{d} r \mathrm{~d} x \\
x_{c}=\frac{1}{\Gamma} \int_{A_{C}} x \omega_{\theta} \mathrm{d} r \mathrm{~d} x, \quad r_{c}=\frac{1}{\Gamma} \int_{A_{C}} r \omega_{\theta} \mathrm{d} r \mathrm{~d} x
\end{gathered}
$$

where $A_{C}$ is the domain within the contour at $20 \mathrm{~s}^{-1}$.

The centroid locations determined using this procedure for $L / D=2.0$ of the NS2 ramps are shown in Fig. 9 for several values of $S r_{L}$. (Each frame shows all of the data obtained from one extendedlength run at the indicated $S r_{L}$.) The centroid locations are indicated by the symbols and the dimensionless circulation, $\Gamma^{*} \equiv \Gamma / U_{\max } D$, associated with each vortex is indicated by the symbol color.

For $S r_{L}=0.13$, the centroid locations are associated with the leading vortex rings as indicated by the relatively large circulation values. In this case, the ring centroids initially move out radially during ring formation near the nozzle and then remain at a constant diameter as they move downstream. The symbol colors indicate that the circulation of the formed vortex rings remains approximately constant as they move downstream in agreement with Kelvin's circulation theorem. It also appears that the centroids drift slightly upward as they move downstream. The source of this drift is uncertain, but it could be due to the small (a few centimeters per second) convection currents in the tank.

For $S r_{L}=0.54$, shown in Fig. 9b, the ring centroids also initially move out radially, but the radial growth seems to be more rapid (being completed at $x / D \approx 0.2$ instead of at $x / D \approx 0.35$ as with $S r_{L}=0.13$ ). After the initial growth, the ring radius contracts slightly over the range $0.4<x / D<4$, again in contrast with 


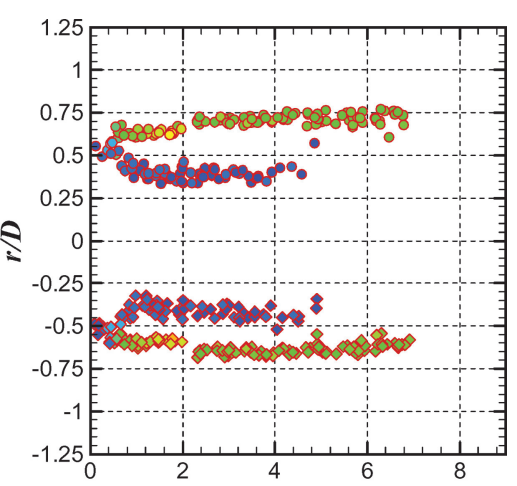

a)

$\boldsymbol{x} / \boldsymbol{D}$

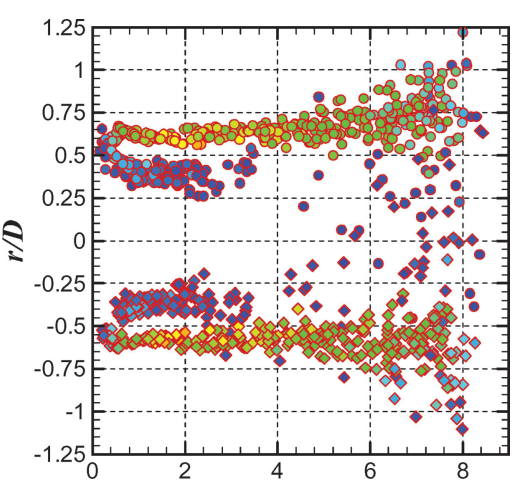

b)

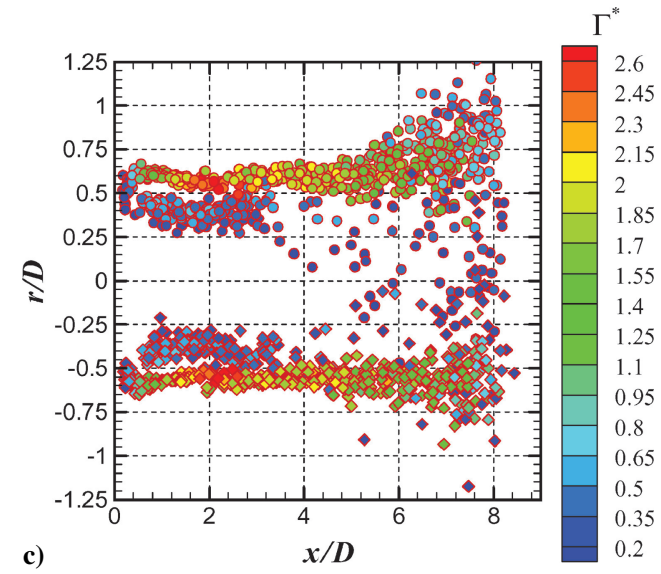

Fig. 11 Centroid locations for $L / D=4.0, \mathrm{NS}$ ramp: $S r_{L}=$ a) 0.06, b) 0.51 , and c) 0.76 ; circulation is made dimensionless according to $\Gamma^{*} \equiv \Gamma /\left(U_{\text {max }} D\right)$.

$S r_{L}=0.13$, which holds a nearly constant radius after the initial formation. These differences in behavior at increased $S r_{L}$ are suggestive of an effect from trailing jet vorticity on initial vortex ring formation and subsequent ring development as proposed earlier in association with the initial reduction in $F_{\mathrm{IJ}}$ as $S r_{L}$ is increased.

Figure $9 \mathrm{~b}$ also shows that the centroids do not follow a consistent path for $x / D>4$, as seen by the wider radial spread of points in this region as compared to Fig. 9a. An apparent decrease in ring circulation accompanies the wider spread of points for $x / D>4$. These clues reflect the tendency for vortex rings to wander off axis as their separation decreases. The wandering or tilting tendency is an inherent instability of a train of vortex rings. Specifically, an off-axis ring is tilted by the induction of neighboring rings in such a way that it continues to move farther off axis. This instability was exploited by helical forcing of pulsed jets in the experiments of Reynolds et al. ${ }^{16}$ to create bifurcating or blooming jets with highly augmented spreading and mixing rates. The present results indicate that such spreading is present even with nominally zero helical forcing.

At the highest $S r_{L}$ shown in Fig. 9, the vortex rings not only wander off axis at larger $x / D$, but they also tend to break up. The evidence for this is seen in the much larger spread in centroid locations for $x / D>4$ and in the appearance of many vortices with low circulation values (less than 0.65 ) in the same region. The low circulation vortices are from vorticity that has broken off from the primary vortices. (Perhaps "eddy" is a better term for these features.) Additionally, the primary vortices themselves are no longer axisymmetric beyond $x / D=4$ and may merge with (or shed vorticity that merges with) other vortices, as evidenced by the points with large circulation (greater than 1.35) in Fig. 9c. The physical mechanism responsible for the apparent breakup of the vortex rings is not obvious from the data, but the complex evolution of the jet vorticity at these conditions makes a model of the jet based on equally spaced, coherent vortex rings untenable for large $S r_{L}$.

For comparison with the $L / D=2.0$ cases just discussed, vortex centroid locations for $L / D=4.0$ of the NS ramp family are shown in Fig. 11. In Fig. 11 two clusters of points are discernible near the nozzle. The inner cluster (with lower circulation) is due to the trailing jets, whereas the outer cluster is from the leading vortex rings. After pinch off occurs, that is, after the circulation of points associated with the leading vortex rings suddenly drops, the points in the inner cluster stop. This indicates, as noted earlier, that trailing jets remain near the nozzle because the convection velocity associated with their vorticity is low. Indeed, the points associated with the trailing jets remain at $x / D<4.5$.

The basic conclusions about the evolution of the vortex rings made for the $L / D=2.0$ cases also appear to be generally true for the leading vortex rings in the $L / D=4.0$ case. The most notable differences are that the centroids of the leading vortex rings appear to wander a bit more for $x / D>4$ than in the $L / D=2.0$ case, that is, the spread in centroid locations around the mean path is larger. Additionally, some breakup of the leading vortices appears at lower
$S r_{L}$ for the $L / D=4.0$ results. This is apparent from the distribution of low circulation vortices near the centerline for $x / D>4$ in Fig. 11b. These effects are probably due to interaction of the leading vortex rings with the trailing jets of previous pulses.

We now return to a discussion of the breakup of the vortex rings that appears as $S r_{L}$ increases. The breakup is quite remarkable. The entire character of the jet changes from being pulse dominated with prominent vortical structures to a much more disordered structure. In fact, vortex disintegration is a more accurate description of the transition. The dramatic nature of the breakup is difficult to discern from Figs. 9 and 11 because they represent only the centroids of regions of vorticity and do not indicate how the vorticity is distributed within the contour used to determine the centroid locations. To illustrate the nature of the breakup and to characterize its effect on the jet structure, we consider the evolution of the half-width of the jet.

The jet half-width $b(x)$ was determined from time-averaged DPIV data of the jet velocity field as the radius where the jet velocity was one-half of the maximum velocity at a given downstream location. The results for $L / D=2.0$ of the NS2 ramps are shown in Fig. 12a. For this low $L / D$ case, the jet diameter is nearly constant for $x / D>1.0$ at $S r_{L}<0.25$. Such behavior is characteristic of a jet dominated by coherent vortical structures. At the slightly higher frequency of $S r_{L}=0.54$, the jet width shows some spreading at $x / D>6.0$, which is due to the vortex ring wandering described in Fig. 9b. For $S r_{L}>0.8$, however, the growth rate of the jet, $\mathrm{d} b / \mathrm{d} x$, changes suddenly from almost zero for $x / D$ less than about 5.0 to a rather large positive value for $x / D>5.0$. The large $\mathrm{d} b / \mathrm{d} x$ for $x / D>5.0$ reflects the disintegration of the vortex rings as the jet flow becomes highly disordered, whereas the sudden change in $\mathrm{d} b / \mathrm{d} x$ reflects how rapidly the disintegration takes place.

By way of contrast, the features for $L / D=2.0$ of the NS2 ramps are largely absent from the $L / D=4.0$ case of the NS ramps, as shown in Fig. 12b. Instead, there is a gradual growth of the jet for $1.0<x / D<8$.0. Thus, for the limited $S r_{L}$ range shown (given that lower $S r_{L}$ runs did not have sufficient data to provide good timeaveraged velocities fields), the presence of a trailing jet in each pulse gives a time-averaged jet character that is not dominated by the leading vortex rings (for which $\mathrm{d} b / \mathrm{d} x$ is negligible) but also does not exhibit a dramatic shift in jet growth rate at large $S r_{L}$. Even though the leading vortex rings in Fig. 11 appeared to behave similarly to the vortex rings in Fig. 9, the presence of the trailing jet modifies the time-averaged character of the jet such that the leading vortex rings do not dominate the flow.

Because most of the interesting behavior for the $L / D=2.0$ case of the NS2 ramps occurs for $x / D>6.0$, the (average) jet growth rates $\mathrm{d} b / \mathrm{d} x$ are evaluated for $9.0>x / D>6.0$ and plotted in Fig. 13 for all cases where DPIV data were collected. For the $L / D<F$ cases (solid symbols), Fig. 13 shows three regions of behavior. In region $1\left(S r_{L}<0.22\right)$, no discernible jet growth is observed for $x / D>6.0$. In region $2\left(0.22<S r_{L}<0.75\right)$, moderate growth rates 


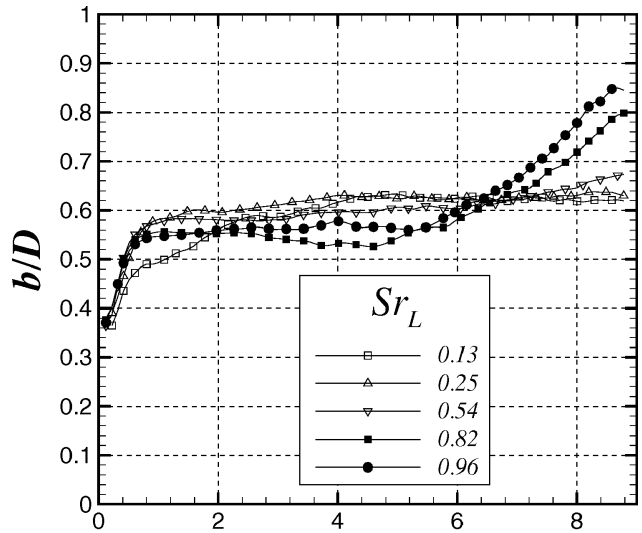

a)

$\boldsymbol{x} / \boldsymbol{D}$

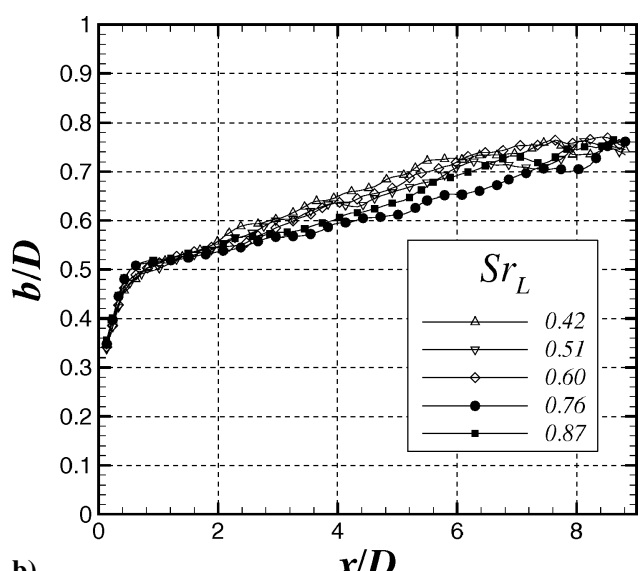

b)

$x / D$

Fig. 12 Downstream evolution of the jet half-width for a) $L / D=2.0$, NS2 ramps; and b) $L / D=4.0$, NS ramps.

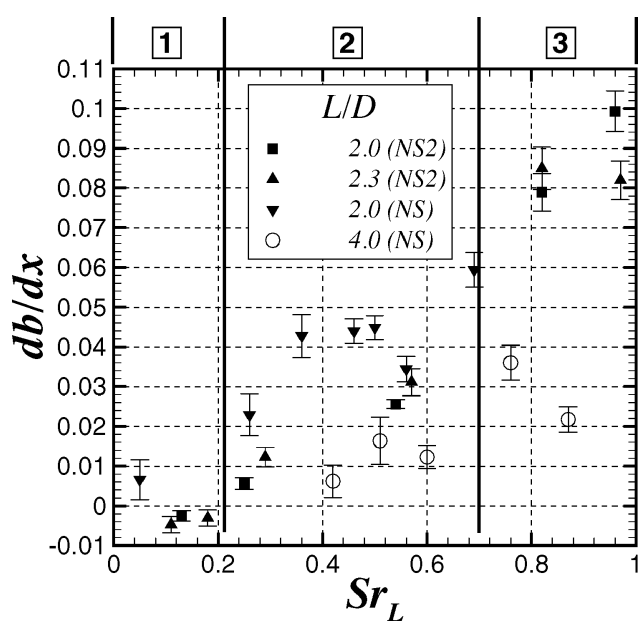

Fig. 13 Jet growth rates for $6.0<x / D<9.0$.

in the range from 0.01 to 0.05 are observed due to vortex wandering. Generally, the growth rates seem to increase with $S r_{L}$ in this region because the induced effect of vortex rings on one another increases as their separation decreases. Finally, in region $3\left(S r_{L}>0.75\right)$, the growth rate jumps suddenly to the values in the range from 0.08 to 0.10 . These growth rates are very similar to that for fully developed (unpulsed) turbulent jets, ${ }^{17,18}$ indicative of the breakup of the vortex rings for $x / D>6.0$ at high $S r_{L}$. Notably, achieving turbulent jet growth rates this close to the nozzle in a fully pulsed jet differs from the results of Bremhorst and Hollis, ${ }^{4}$ who found that the flow in their fully pulsed jet was pulse dominated for 50 diameters downstream of the nozzle and approached the character of a more traditional turbulent jet thereafter. However, their results were for $S r_{L}=0.33$ and $L / D>142$, and so the strong vortex ring interactions apparent in the present study could not be observed.

By way of comparison, the growth rates for the $L / D=4.0$ results of the NS ramps are shown as the open symbols in Fig. 13. Generally, the growth rates for this case are much lower and do not follow the trends observed for the $L / D<F$ cases, as anticipated from Fig. 12 .

The jet width measurements demonstrate that even in fully pulsed jets, the flow can transition from a pulse-dominated character to a more turbulent (or transitional) character as early as five diameters from the nozzle exit plane for $S r_{L}>0.75$ (and $L / D<F$ ). This, combined with the vortex wandering that occurs for $S r_{L}$ as low as 0.25 , underscores the pitfalls of modeling a fully pulsed jet as a train of coaxial vortex rings. Whereas such a model is appealing because it explicitly includes the nature of the jet unsteadiness, it seems to be appropriate only for small $L / D$ at low $S r_{L}$. In this region, however, weak trailing jets left behind by preceding pulses can have a more significant effect on performance than the interaction of the main vortex rings. Hence, modeling the propulsive performance of a fully pulsed jet presents significant challenges because the actual vortex evolution in the jet is not simple but plays a crucial role in the jet performance.

\section{Conclusions}

Thrust augmentation in a fully pulsed jet was investigated experimentally by direct measurement of the time-averaged thrust as a function of dimensionless pulse size $L / D$ and dimensionless frequency (or duty cycle) $S r_{L}$. Significant augmentation was observed over the entire parameter range tested, both in terms of thrust compared to an equivalent steady jet with identical mass flux, that is, $F_{\mathrm{SJ}}>1$, and in terms of thrust compared to an equivalent intermittent jet where vortex ring formation was ignored, that is, $F_{\mathrm{IJ}}>1$. In particular, $F_{\mathrm{SJ}}$ as high as 1.90 (90\% thrust augmentation) was observed for $L / D=2$ (small enough to avoid vortex ring pinch off) and $S r_{L}$ approaching 1.0 (with larger $F_{\mathrm{SJ}}$ at lower $S r_{L}$ ). This is substantially greater than the augmentation observed for low-amplitude forcing, indicating the benefits of a highly pulsed jet. Additionally, if an ejector were included, the results of Wilson and Paxon ${ }^{7}$ suggest that an additional 33\% augmentation could be achieved, giving a net $153 \%$ thrust augmentation over a steady jet of equivalent mass flux $\left(F_{\mathrm{SJ}}=2.53\right)$ for $S r_{L}$ approaching 1.0 at small $L / D$. The present investigation was limited to $L / D \geq 2$ because the large contraction between the plenum and the nozzle made it difficult to produce repeatable pulses at $L / D<2$. It would be useful to investigate thrust augmentation at $L / D<2$, however, because augmentation seemed to be greatest at small $L / D$.

The $F_{\mathrm{IJ}}$ results, on the other hand, highlighted the role played by nozzle exit overpressure in thrust augmentation. Because overpressure is developed during vortex ring formation, the dynamics of the formation process strongly influenced $F_{\mathrm{IJ}}$. Specifically, $F_{\mathrm{IJ}}$ was lower for $L / D$ large enough to observe pinch off, that is, $L / D>F$, because a trailing jet contributes negligible overpressure. Also, as $S r_{L}$ increased for a constant $L / D$, a rather sharp decrease in $F_{\mathrm{IJ}}$ was observed at low $S r_{L}$, followed by a more gradual decrease as $S r_{L}$ increased toward 1.0. The first decline in $F_{\mathrm{IJ}}$ was attributed to the presence of remnants from preceding pulses near the nozzle, that is, trailing jets, that interacted with the forming rings. Two mechanisms were proposed by which this interaction could decrease $F_{\mathrm{IJ}}$, but only indirect verification of their effect was possible, and more detailed data during the initial ring formation would be helpful in quantifying the role of each. The more gradual decrease in $F_{\mathrm{IJ}}$ at higher $S r_{L}$ was attributed to an interaction between entire pulses. As $S r_{L}$ increases, the vorticity from preceding pulses is closer to the nozzle at the ejection of the each pulse, which requires less fluid to be accelerated by the issuing pulse and reduces nozzle exit overpressure.

Because the interaction of vortical structures seemed to play a key role in thrust augmentation, the global structure of the jet was considered in more detail using DPIV measurements of the flow velocity and azimuthal vorticity for $0<x / D<9$. Although a clear train of coaxial vortex rings was observed for $L / D<F$ and $S r_{L}<0.22$, the vortex rings tended to wander off axis for $0.22<S r_{L}<0.75$ 
(with increasing severity for higher $S r_{L}$ ) and then to break up entirely as $S r_{L}$ increased above 0.75 . Observations of the growth rates of the jet half-width provided similar conclusions. Specifically, for $S r_{L}>0.75$ and $L / D$ approximately 2.0 , the jet width changed suddenly at $x / D \approx 5.0$ from negligible growth to a growth rate comparable to a fully developed (unpulsed) turbulent jet. Taken together, these observations exclude a simple model of the jet based on a train of coaxial vortex rings. On the other hand, they may help explain the decrease in jet penetration depth observed by Johari et al. ${ }^{6}$ for fully pulsed jets in crossflow as the duty cycle (equivalently, $S r_{L}$ ) increased because more coherent vortical structures would tend to penetrate the crossflow more directly and mix less effectively.

As for the larger $L / D$ cases, the presence of trailing jets in the $L / D=4.0$ results significantly affected the evolution of the leading vortex rings and the jet as a whole, leading to a small but steady growth in the jet half-width over the entire $1<x / D<9$ range for $S r_{L}>0.4$. Clearly, the evolution of the flow is complex and presents significant challenges for developing a model of thrust augmentation in fully pulsed jets.

\section{Acknowledgment}

The authors gratefully acknowledge the support of the National Science Foundation for this work.

\section{References}

${ }^{1}$ Crow, S. C., and Champagne, F. H., "Orderly Structure in Jet Turbulence," Journal of Fluid Mechanics, Vol. 48, 1971, pp. 547-591.

${ }^{2}$ Vermeulen, P. J., Ramesh, V., and Yu, W. K., "Measurements of Entrainment by Acoustically Pulsed Axisymmetric Air Jets," Journal of Engineering for Gas Turbines and Power, Vol. 108, No. 3, 1986, pp. 479-484.

${ }^{3}$ Vermeulen, P. J., Rainville, P., and Ramesh, V., "Measurements of the Entrainment Coefficient of Acoustically Pulsed Axisymmetric Free Air Jets," Journal of Engineering for Gas Turbines and Power, Vol. 114, No. 2, 1992, pp. 409-415.

${ }^{4}$ Bremhorst, K., and Hollis, P. G., "Velocity Field of an Axisymmetric Pulsed, Subsonic Air Jet," AIAA Journal, Vol. 28, No. 12, 1990, pp. 2043-2049.

${ }^{5}$ Bremhorst, K., and Gehrke, P. J., "Measured Reynolds Stress Distribu- tions and Energy Budgets of a Fully Pulsed Round Air Jet," Experiments in Fluids, Vol. 28, No. 6, 2000, pp. 519-531.

${ }^{6}$ Johari, H., Pacheco-Tougas, M., and Hermanson, J. C., "Penetration and Mixing of Fully Modulated Turbulent Jets in Crossflow," AIAA Journal, Vol. 37, No. 7, 1999, pp. 842-850.

${ }^{7}$ Wilson, J., and Paxson, D. E., "Unsteady Ejector Performance: An Experimental Investigation Using a Resonance Tube Driver," AIAA Paper 2002-3632, July 2002.

${ }^{8}$ Sarohia, V., Bernal, L., and Bui, T., "Entrainment and Thrust Augmentation in Pulsatile Ejector Flows," Jet Propulsion Lab., California Inst. of Technology, Rept. 81-36, Pasadena, CA, Aug. 1981.

${ }^{9}$ Didden, N., "On the Formation of Vortex Rings: Rolling-up and Production of Circulation," Journal of Applied Mathematics and Physics (ZAMP), Vol. 30, No. 1, 1979, pp. 101-116.

${ }^{10}$ Krueger, P. S., and Gharib, M., "The Significance of Vortex Ring Formation to the Impulse and Thrust of a Starting Jet," Physics of Fluids, Vol. 15, No. 5, 2003, pp. 1271-1281.

${ }^{11}$ Gharib, M., Rambod, E., and Shariff, K., "A Universal Time Scale for Vortex Ring Formation," Journal of Fluid Mechanics, Vol. 360, 1998, pp. 121-140.

${ }^{12}$ Willert, C. E., and Gharib, M., "Digital Particle Image Velocimetry," Experiments in Fluids, Vol. 10, No. 4, 1991, pp. 181-193.

${ }^{13}$ Westerweel, J., Dabiri, D., and Gharib, M., "The Effect of a Discrete Window Offset on the Accuracy of Cross-Correlation Analysis of Digital PIV Recordings," Experiments in Fluids, Vol. 23, No. 1, 1997, pp. 20-28.

${ }^{14}$ Rosenfeld, M., Rambod, E., and Gharib, M., "Circulation and Formation Number of Laminar Vortex Rings," Journal of Fluid Mechanics, Vol. 376, 1998, pp. 297-318.

${ }^{15}$ Weihs, D., "Periodic Jet Propulsion of Aquatic Creatures," Fortschritte der Zoologie, Vol. 24, No. 2/3, 1977, pp. 171-175.

${ }^{16}$ Reynolds, W. C., Parekh, D. E., Juvet, P. J. D., and Lee, M. J. D., "Bifurcating and Blooming Jets," Annual Review of Fluid Mechanics, Vol. 35, 2003, pp. 295-315.

${ }^{17}$ Wygnanski, I., and Fiedler, H., "Some Measurements in the SelfPreserving Jet," Journal of Fluid Mechanics, Vol. 38, 1969, pp. 577-612.

${ }^{18}$ Hussein, H. J., Capp, S. P., and George, W. K., "Velocity Measurements in a High-Reynolds-Number, Momentum-Conserving, Axisymmetric, Turbulent Jet," Journal of Fluid Mechanics, Vol. 258, 1994, pp. 31-75.

A. Karagozian Associate Editor 